OPEN ACCESS

Edited by:

Sergio Davinelli,

University of Molise, Italy

Reviewed by:

Anthony Hiu King Tsang,

University of Cambridge,

United Kingdom

Etienne Challet,

Université de Strasbourg, France

*Correspondence:

Daniela A. Koppold-Liebscher daniela.liebscher@charite.de

Specialty section:

This article was submitted to Clinical Nutrition,

a section of the journal

Frontiers in Nutrition

Received: 01 February 2021

Accepted: 21 June 2021

Published: 29 July 2021

Citation:

Koppold-Liebscher DA, Klatte C,

Demmrich S, Schwarz J, Kandil FI,

Steckhan N, Ring R, Kessler CS,

Jeitler $M$, Koller $B$

Ananthasubramaniam $B$,

Eisenmann C, Mähler A,

Boschmann M, Kramer A and

Michalsen A (2021) Effects of Daytime

Dry Fasting on Hydration, Glucose

Metabolism and Circadian Phase: A

Prospective Exploratory Cohort Study

in Bahá'i Volunteers.

Front. Nutr. 8:662310.

doi: 10.3389/fnut.2021.662310

\section{Effects of Daytime Dry Fasting on Hydration, Glucose Metabolism and Circadian Phase: A Prospective Exploratory Cohort Study in Bahá'í Volunteers}

Daniela A. Koppold-Liebscher ${ }^{1,2 *}$, Caroline Klatte ${ }^{1}$, Sarah Demmrich ${ }^{3}$, Julia Schwarz ${ }^{4}$, Farid I. Kandil ${ }^{1,5}$, Nico Steckhan ${ }^{1,6}$, Raphaela Ring ${ }^{1}$, Christian S. Kessler ${ }^{1,2}$, Michael Jeitler ${ }^{1,2}$, Barbara Koller ${ }^{7}$, Bharath Ananthasubramaniam ${ }^{8}$, Clemens Eisenmann ${ }^{9}$, Anja Mähler ${ }^{10,11}$, Michael Boschmann ${ }^{10}$, Achim Kramer $^{7}$ and Andreas Michalsen ${ }^{1,2}$

\footnotetext{
${ }^{1}$ Institute of Social Medicine, Epidemiology and Health Economics, Charité-Universitätsmedizin Berlin, Corporate Member of Freie Universität Berlin, Humboldt-Universität zu Berlin, and Berlin Institute of Health, Berlin, Germany, ${ }^{2}$ Department of Internal and Integrative Medicine, Immanuel Hospital Berlin, Berlin, Germany, ${ }^{3}$ Department of Sociology, Cluster of Excellence Religion and Politics, University of Münster, Münster, Germany, ${ }^{4}$ Department of Oecotrophology, Hochschule Niederrhein, University of Applied Science, Mönchengladbach, Germany, ${ }^{5}$ Department of Neurology, Campus Benjamin Franklin, Charité Universitätsmedizin Berlin, Berlin, Germany, ${ }^{6}$ Connected Healthcare, Hasso Plattner Institute, University of Potsdam, Potsdam, Germany, ${ }^{7}$ Laboratory of Chronobiology, Institute of Medical Immunology, Charité-Universitätsmedizin Berlin, Corporate Member of Freie Universität Berlin, Humboldt-Universität zu Berlin, and Berlin Institute of Health, Berlin, Germany, ${ }^{8}$ Institute for Theoretical Biology, Charité Universitätsmedizin Berlin, Berlin, Germany, ${ }^{9}$ Department of Sociology, University of Konstanz, Konstanz, Germany, ${ }^{10}$ Experimental and Clinical Research Center-a Joint Cooperation Between Charité-Universitätsmedizin Berlin and Max Delbrueck Center for Molecular Medicine, Berlin, Germany, ${ }^{11}$ NeuroCure Clinical Research Center, Charité-Universitätsmedizin Berlin, Berlin, Germany
}

Background: Religiously motivated Bahá'í fasting (BF) is a form of intermittent dry fasting celebrated by abstaining from food and drinks during daylight hours every year in March for 19 consecutive days.

Aim: To test the safety and effects of BF on hydration, metabolism, and the circadian clock.

Methods: Thirty-four healthy Bahá'í volunteers (15 women) participated in this prospective, exploratory cohort study. Laboratory examinations were carried out in four study visits: before fasting (V0), in the third week of fasting (V1) as well as 3 weeks (V3) and 3 months (V4) after fasting. Data collection included blood and urine samples, anthropometric measurements and bioelectrical impedance analysis. At V0 and V1, 24and 12-hour urine and serum osmolality were measured. At V0-V2, alterations in the circadian clock phase were monitored in 16 participants. Our study was augmented by an additional survey with 144 healthy Bahá'í volunteers filling out questionnaires and with subgroups attending metabolic measurements $(n=11)$ and qualitative interviews $(n=$ 13), the results of which will be published separately.

Results: Exploratory data analysis revealed that serum osmolality $(n=34, p<0.001)$ and 24-hour urine osmolality $(n=34, p=0.003)$ decreased during daytime fasting but remained largely within the physiological range and returned to pre-fasting levels during 
night hours. BMI (body mass index), total body fat mass, and resting metabolic rate decreased during fasting $(n=34, p<0.001)$, while body cell mass and body water appeared unchanged. The circadian phase estimated by transcript biomarkers of blood monocytes advanced by $1.1 \mathrm{~h}(n=16, p<0.005)$ during fasting and returned to pre-fasting values 3 weeks after fasting. Most observed changes were not detectable anymore 3 months after fasting.

Conclusions: Results indicate that BF (Bahá'i fasting) is safe, has no negative effects on hydration, can improve fat metabolism and can cause transient phase shifts of circadian rhythms.

Trial Registration: https://www.clinicaltrials.gov/, identifier: NCT03443739.

Keywords: hydration, religious, intermittent fasting, chronobiology, water deprivation, time-restricted eating, fasting, diurnal fasting

\section{INTRODUCTION}

Fasting with its preventive and therapeutic effects has, in general, been explored more and more over the past decade (1-10). However, dry fasting (DF) has not received as much attention in the scientific community. Most studies in this regard have focused on the Ramadan fast $(11,12)$, not specifically exploring DF as such, while serious consequences of a dysregulated body fluid balance are clear for every clinician (13), making studies on DF difficult to conduct.

However, Ramadan fasting is a complex model for DF, as the daily duration of fasting varies greatly over the years (from 11 to 22 hours a day, depending on the season and geographic location) (14). Apart from studies on Ramadan fasting, only a few studies have focused on DF itself (15-20). From these, only Papagiannopoulos et al. and Papagiannopoulos-Vatopaidinos et al. $(15,20)$ have focused on the physiology of prolonged DF, examining 10 participants each for 5 consecutive days of water and food deprivation.

The Baháí religion is monotheistic and was founded by Baháu'lláh (1817-1892) (21). Fasting is seen by Baháís as one of the most significant spiritual duties of a healthy individual (22). The sick, the elderly, as well as children under 15 years of age and pregnant, menstruating, or nursing women are exempt from the religious duty to fast (22). Worldwide, the followers of the Bahái 1 religion fast every year in March, abstaining from food and drink from sunrise to sunset for 19 consecutive days (22). This practice could best be described as an intermittent dry fast. No additional dietary regulations exist for believers during the fasting period or in general, except that consumption of alcoholic beverages is

Abbreviations: BIA, bioelectrical impedance analysis; BGA, blood gas analysis; $\mathrm{BMI}$, body mass index; $\mathrm{BF}$, Baháí fasting; $\mathrm{BQ}$, participants of the large-scale study with questionnaires; $\mathrm{CB}$, participants of chronobiological measurements; DC, Department of Chronobiology; DF, dry fasting; DLMO, dim light melatonin onset; ECRC, experimental and clinical research center; HbAlc, glycosylated hemoglobin; IDF, intermittent dry fasting; IM, Department of Integrative Medicine under the Institute of Social Medicine, Epidemiology and Health Economics; PP, participants of laboratory and anthropometric measurements; V0, V1, V2, and V3, study visits with laboratory examinations; VQ1, VQ2, VQ3, and VQ4, study visits with questionnaires. prohibited throughout the year. In contrast to Ramadan fasting, Bahá'i fasting (BF) has a fixed date in the year, is of shorter duration (i.e. 19 days) and, since it takes place in March, fasting time varies only between 10-13 hours daily. At the study site in Berlin/Germany, the exact duration is 10.90 hours at the beginning and 12.12 hours at the end of the fasting period. This makes it a good model for exploring the effects of intermittent dry fasting (IDF) on healthy adults, as no relevant changes occur in climate or timing when comparing data from different years or places. Since research has suggested that Ramadan fasting causes alterations of normal circadian rhythms (23), we also explored the effects of BF on the circadian phase (chronotype).

Thus, in this exploratory study, fluid balance in healthy adults during a religiously motivated IDF was assessed. Additionally, it was explored, whether known physiological mechanisms of fasting are also activated through this kind of fasting, which is shorter in duration than other fasting intervals of, for example, time-restricted eating (1) or intermittent fasting (5). More specifically, the aim was to assess urine and serum osmolality, renal and liver function, blood fatty acids, ketones, blood gas analysis, and anthropometric variables, as well as effects on normal daily patterns of eating and drinking. The collected data should serve to generate hypotheses for future studies in the field of IDF in general as well as assessing the safety and effects of BF.

\section{METHODS}

\section{Study Design}

This longitudinal, exploratory cohort study focussed on changes in physiological parameters. It was augmented by a mixedmethods study on Baháí fasting.

This study represents a quasi-experiment with a longitudinal observational design (24). As fasting time and duration are fixed for all Baháís by religious provisions, there was no possibility to randomize the volunteers into a fasting and a non- (or delayed-) fasting group. Using a control group with a nonreligious motivation could lead to a bias, as religiously motivated people may have higher compliance rates to dietary norms as well as engage in different health-related behaviors compared 


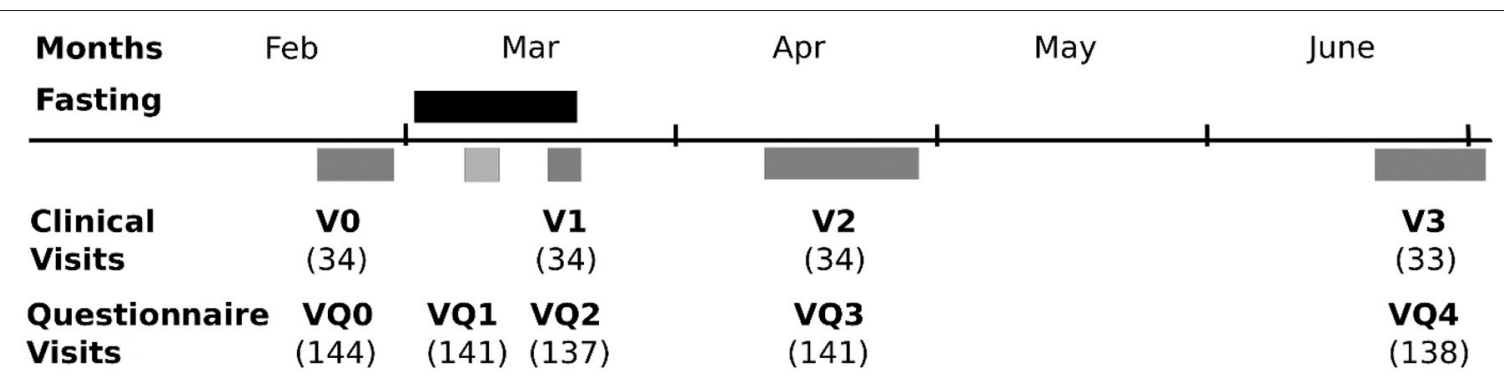

FIGURE 1 | A flow chart of visits with participant numbers.

with non-adherents (25). The external laboratory personnel, as well as the statistician, were blinded. All other study personnel were involved directly with the study participants during visits. Therefore, no blinding was possible in this case.

The study protocol was approved by the institutional review board of Charité Universitätsmedizin Berlin (Charitéplatz 1, 10117 Berlin) in January 2018 (ID: EA4/216/17), was registered with ClinicalTrials.gov (ID: NCT03443739), and carried out according to the standards of the Declaration of Helsinki. Written informed consent was obtained from all the participants prior to study entry.

\section{Setting}

Three departments of the same university (Charité Universitätsmedizin Berlin) cooperated for this study: the Department of Integrative Medicine at the Institute of Social Medicine, Epidemiology and Health Economics (IM), the Experimental and Clinical Research Center (ECRC), and the Department of Chronobiology (DC). The two latter departments conducted sub-studies. The main study site was at the Department of Integrative Medicine. Physiological data comprising urine and blood samples, as well as anthropometric measurements, were acquired at the four in-house visits in the Department of Integrative Medicine at the Institute of Social Medicine, Epidemiology and Health Economics (IM) or, for a subgroup, at the Experimental and Clinical Research Center (ECRC). The visits were conducted in the 2 weeks before the beginning of the fasting period (V0), in the last week of the fast (V1), 3 weeks after the end of the fast (V2), and finally, 3 months after the end of the fasting period (V3). Figure 1 shows these visits along with the additional other visits for the psychometric questionnaires of the companion study and the other sub-studies.

\section{Participants}

Original first recruitment was conducted via the National Spiritual Assembly of the Bahá ís of Germany (23), who spread the information via email to all Bahá'ís in Germany. For the aims of this study, 172 healthy volunteers were screened, and 144 were considered eligible (see below) for the questionnaire survey and were enclosed between January and February 2018. All eligible individuals filled out electronic questionnaires on subjective physical and psychological effects of Baháí fasting (group "BQ").

Of these, all the participants living in the wider region of Berlin were invited to participate in the additional laboratory tests reported upon here. Thirty-four subjects could be enclosed in this sub-study with its tests for physiological parameters (group "PP"), giving their informed written consent. Again, 17 of these 34 participants agreed to donate additional blood samples for chronobiological measurements and to answer additional questionnaires (group "CB"). The results of the psychometric questionnaires, as well as those of individual in-depth and focus group interviews and another subgroup concentrating on metabolic responses, will be reported separately.

\section{Eligibility Criteria}

The participants were screened at the IM and then referred to all sub-studies.

Inclusion criteria were registered membership in the Bahá'í community, planned adherence to fasting in the upcoming fasting period and age between 18 and 69 years (children and elderly are exempt from the religious duty to fast, and, because of the necessity for informed consent, no youth between 15 and 17 years were recruited for the study).

Exclusion criteria were: scheduled interruption of the fast for more than 5 days (for e.g., due to planned travel), pregnancy or breastfeeding, serious physical, or psychological illness, known eating disorder, participation in another study, no email address (because of electronic questionnaires). Individuals who worked in shifts and planned long-distance travel shortly before and during the study period were also excluded from eligibility for the chronobiological measurements.

\section{Outcomes/Variables}

Primary outcome measures were the hydration status of fasting individuals and changes in serum osmolality and urine osmolality in 12- and 24-hour-urine samples. Secondary outcome measures were anthropometric parameters, such as body weight, BMI, body composition via bioelectrical impedance analysis (BIA, measured by the octapolar BIACORPUS RX 4004M ${ }^{\circledR}$ ) and the waist-to-hip ratio. The resting metabolic rate was calculated by an algorithm implemented in the BIA software provided by the manufacturer. Blood pressure, heart rate, and standard blood count were assessed alongside metabolic parameters, such as 
ketone bodies in capillary blood (on the spot, with ACCUCHEK $^{\circledR}$ device) and urine, blood glucose levels, glycosylated hemoglobin (HbAlc), fructosamine, C-reactive protein, liver enzymes, and serum cholesterol. Additionally, the fluid balance was assessed by kidney parameters, 12- and 24-hour urine creatinine clearance, serum electrolytes, acid-based balance measured by blood gas analysis (on the spot, with the ABL80 FLEX ${ }^{\circledR}$ blood gas analyser), 24-hour and spontaneous urine osmolality, 24-hour urine specific gravity, and cystatin C. All laboratory parameters were measured at Labor Berlin, unless otherwise indicated. As most laboratory measurements had to be carried out in a fasting state, they were carried out between 7:30 a.m. and 11:00 a.m. outside of the fasting period, while, during $\mathrm{BF}$, as most subjects would have breakfast before sunrise, the measurements were done between 4:30 p.m. and 6:30 p.m., if not otherwise indicated.

In group $\mathrm{CB}$ (17 subjects), an additional blood sample was taken between $\sim 8$ a.m. and $\sim 10$ a.m. at V0, V1, and V2 to assess the circadian phase (chronotype) (Supplementary Table 1) (26). Note that the determination of the circadian phase with the BodyTime assay is independent of the time of sample collection (26). Briefly, monocytes were sorted from whole blood, using magnetic cell sorting, total RNA was prepared, and the expression of 24 biomarker genes was analyzed, using NanoString technology. Based on these data, the body time algorithm allowed the prediction of the circadian phase. In this subgroup, the validated Munich Chronotype questionnaire (27) was also used.

Timing of the measurements constituted a challenge. Due to capacity constraints of the study centers, measurements could not all be done at the same time. Therefore, the measurements had to be spread over the last week of $\mathrm{BF}$ and start 2 hours before breaking the fast for dinner so that the mean fasting time for our measurements was 17 fasting days and 11 hours of daily fasting duration.

For a better overview of the whole study setting, we also mention the following measurements, the results of which will be published separately: Body composition was measured by airdisplacement plethysmography, parameters of systemic energy metabolism by indirect calorimetry, and parameters of adipose tissue and skeletal muscle metabolism by microdialysis in two subgroups. Furthermore, validated questionnaires on quality of life, mood, mindfulness, and spirituality were used in electronic form for the BQ group. Additionally, the focus group and individual interviews were carried out by a trained sociologist.

\section{Bias}

Recruitment for both this study and the accompanying ones was based on voluntary participation and may thus have introduced a sampling bias in favor of motivated and health-conscious subjects. Apart from that, the implementation of an interventiongroup-only design is, in so far, justified as the aim of the study was to explore the safety and general health effects of BF, using pre- and post-fasting observations to explore the strength and duration of the fasting effects.

\section{Study Size}

The study was planned as an exploratory study to help generate hypotheses for future studies. All $p$-values lower than
0.10 are regarded as potentially interesting and those below 0.05 as strongly interesting for future studies. The number of the participants $(n=34)$ allows to detect all high-sized and medium-sized effects of $f \geq 0.16$, corresponding to a Cohen's $d \geq 0.32$ (28) under the assumption of standard parameters [alpha $=0.05$, beta $=0.20$ (power of $80 \%$ ) ] and a higher correlation between measures of 0.7 (based on previous in-house data).

\section{Statistical Methods}

As blood parameters are typically not normally distributed, all analyses were conducted, using non-parametric tests. The Friedman test, a counterpart to a one-factorial repeated measures ANOVA, was used to compare the physiological parameters across the four (three) clinical visits, while pairwise posthoc analyses were applied, using the Wilcoxon's signed-rank test. As usual, in exploratory studies, resulting $p$-values are presented as such and not compared against any adjusted alpha value.

\section{Missing Data}

Some physiological data were missing during the laboratory failures and one subject not attending the last visit. The Little test indicated that the missing data were completely at random ("MCAR") (29) and were replaced using multiple imputation methods ("multiple imputer") implemented in the Python's (v. 3.7) SciPy library.

\section{RESULTS}

\section{Participants}

Thirty-four volunteers ( $15 \mathrm{w}, 19 \mathrm{~m}$, age: $41.09 \pm 14.54$ years) practicing $\mathrm{BF}$ and living in or near Berlin participated in the visits and tests of physiological parameters (group "PP"). They had a mean BMI of $25.74 \pm 5.13 \mathrm{~kg} / \mathrm{m}^{2}$, corresponding well to the average BMI of $26 \mathrm{~kg} / \mathrm{m}^{2}$ in Germany (30). Further sociodemographic data and baseline characteristics of these participants are shown in Tables 1, 2, respectively. Confidence intervals of the baseline laboratory parameters are clinically unremarkable.

Of these 34 volunteers, 17 subjects (the "CB" subgroup) donated additional blood samples for chronobiological measurements. One participant was not able to attend the last follow-up visit in person. While part of the visit could be conducted via telephone (questionnaires), no physiological samples could be obtained. These, as well as missing laboratory data from some subjects for some visits due to a laboratory failure, were filled in by multiple imputations (see Methods).

\section{Outcomes}

\section{Primary Outcome: Hydration Markers}

Plasma osmolality changed during fasting $\left[\chi^{2}(3)=19.61, P<\right.$ 0.001]. It decreased from V0 to V1 and increased to baseline (V0) or even higher levels from V1 to V2, returning to baseline levels by V3 (Table 3, Figure 2A). However, all these changes were within the physiological range.

Osmolality of the 24-hour urine (Figure 2B) changed similarly $\left[\chi^{2}(3)=13.91, P<0.001\right]$, with a decrease from V0 to 
TABLE 1 | Sociodemographic characteristics of the participants in the laboratory measurements.

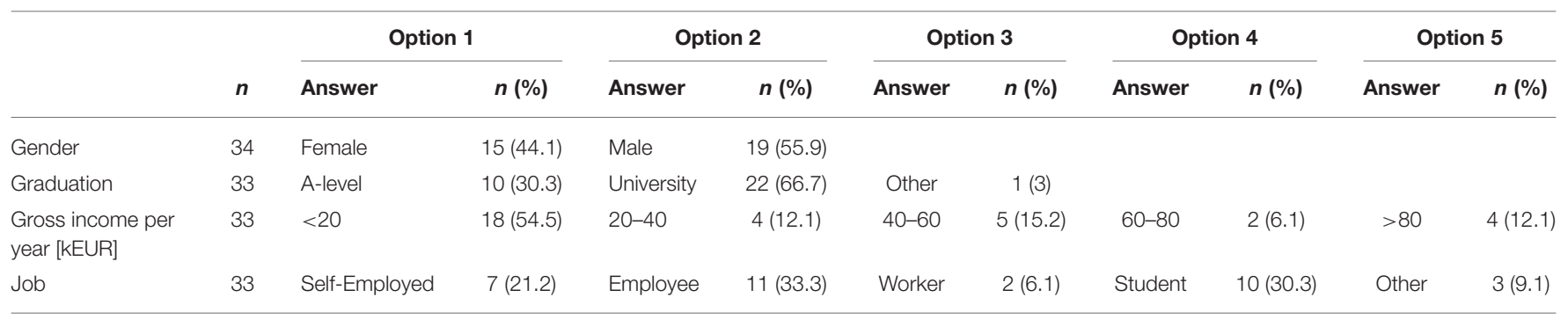

TABLE 2 | Characteristics of participants at baseline (VO).

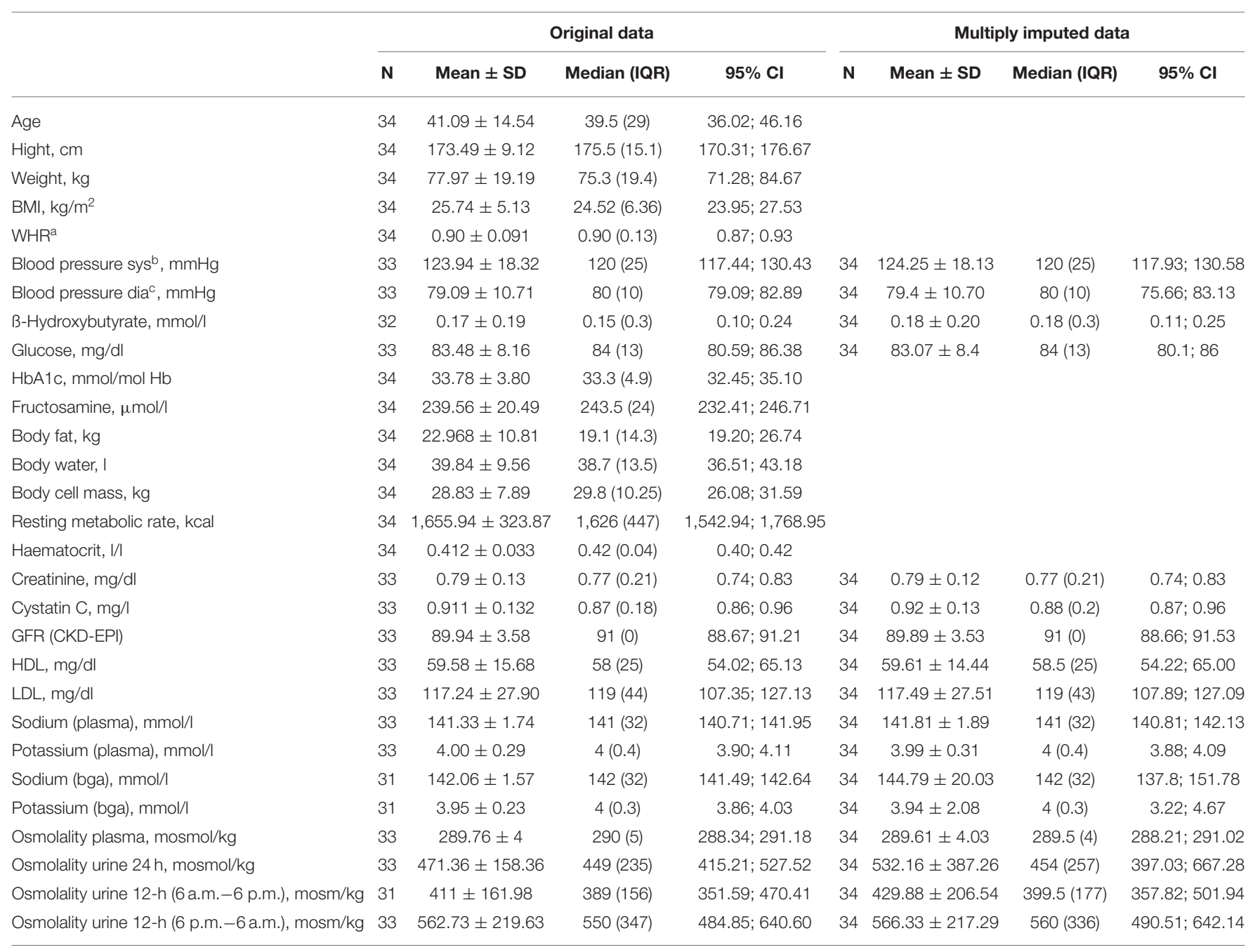

${ }^{a}$ WHR, waist-to-hip ratio; ${ }^{b}$ sys, systolic; ${ }^{c}$ dia, diastolic.

$\mathrm{V} 1$ and a subsequent increase from V1 to V2 to baseline levels. Indepth analysis of the samples, however, revealed that this initial decrease was mainly due to a distinct decrease in nocturnal urine osmolality (sampled from 6 p.m. to 6 a.m.), whereas changes in diurnal urine were less marked. Overall, these changes were largely within the physiological range and, therefore, clinically not relevant.

\section{Kidney Values and Electrolytes}

The glomerular filtration rate, as estimated by the equation of the Chronic Kidney Disease Epidemiology Collaboration (GFR via CKD-EPI), creatinine, and cystatin $C$ did not change relevantly during fasting (GFR: $P=0.584$, creatinine: $p=0.060$, and cystatin C: $p=0.073)$. Creatinine showed a tendency to increase slightly during fasting (V0-V1: $0.03 \pm 0.09 \mathrm{mg} / \mathrm{dl})$ and remained 
TABLE 3 | Anthropometric, blood, and urine parameters before, during, and after 19 days of Bahá'í fasting (BF).

\begin{tabular}{|c|c|c|c|c|c|c|c|c|c|c|c|c|c|c|c|c|c|c|c|}
\hline & \multirow[t]{2}{*}{$\begin{array}{l}\text { Reference } \\
\text { range }\end{array}$} & \multirow[t]{2}{*}{ vo } & \multirow[t]{2}{*}{ V1 } & \multirow[t]{2}{*}{ V2 } & \multirow[t]{2}{*}{ v3 } & \multicolumn{2}{|c|}{$\begin{array}{c}\text { Friedmann } \\
\text { main analysis }\end{array}$} & \multicolumn{2}{|c|}{ V1 vs. V0 } & \multicolumn{2}{|c|}{ V1 vs. V2 } & \multicolumn{2}{|c|}{ V2 vs. V0 } & \multicolumn{2}{|c|}{ V3 vs. V0 } & \multicolumn{2}{|c|}{ V1 vs. V3 } & \multicolumn{2}{|c|}{ V2 vs. V3 } \\
\hline & & & & & & $\chi^{2}(3)$ & $p$ & $z$ & $p$ & $z$ & $p$ & $z$ & $p$ & $z$ & $p$ & $z$ & $p$ & $z$ & $p$ \\
\hline Weight, kg & & $\begin{array}{r}75.3 \\
(19.4)\end{array}$ & $\begin{array}{l}72.75 \\
(21.8)\end{array}$ & $\begin{array}{l}74.2 \\
(20.9)\end{array}$ & $\begin{array}{r}75.8 \\
(18.8)\end{array}$ & 18.027 & $<0.001$ & 4.180 & $<0.001$ & -2.114 & 0.207 & 2.067 & 0.233 & 1.644 & 0.601 & -2.536 & 0.067 & -0.423 & 1.000 \\
\hline $\mathrm{BMI}, \mathrm{kg} / \mathrm{m}^{2}$ & & $\begin{array}{l}24.52 \\
(6.18)\end{array}$ & $\begin{array}{l}24.24 \\
(6.23)\end{array}$ & $\begin{array}{l}24.52 \\
(5.71)\end{array}$ & $\begin{array}{c}24.43 \\
(5.3)\end{array}$ & 17.74 & $<0.001$ & 3.983 & $<0.001$ & 2.359 & $<0.001$ & 2.342 & 0.019 & 2.069 & 0.039 & 1.428 & 0.153 & 0.239 & 0.811 \\
\hline WHR & & $\begin{array}{l}0.895 \\
(0.13)\end{array}$ & $\begin{array}{c}0.92 \\
(0.13)\end{array}$ & $\begin{array}{c}0.88 \\
(0.09)\end{array}$ & $\begin{array}{c}0.87 \\
(0.14)\end{array}$ & 30.526 & $<0.001$ & 0.892 & 1.000 & -2.771 & 0.034 & 1.879 & 0.362 & 4.086 & $<0.001$ & -4.978 & $<0.001$ & -2.207 & 0.164 \\
\hline Body fat, kg & & $\begin{array}{c}19.1 \\
(13.48)\end{array}$ & $\begin{array}{l}19.05 \\
(12.7)\end{array}$ & $\begin{array}{c}19.25 \\
(12.98)\end{array}$ & $\begin{array}{c}18.25 \\
(13.55)\end{array}$ & 31.83 & $<0.001$ & 4.36 & $<0.001$ & 2.248 & 0.025 & 3.359 & 0.001 & 3.915 & $<0.001$ & 0.53 & 0.596 & 1.103 & 0.27 \\
\hline Body water, I & & $\begin{array}{c}38.7 \\
(13.5)\end{array}$ & $\begin{array}{l}38.95 \\
(15.8)\end{array}$ & $\begin{array}{c}38.5 \\
(13.1)\end{array}$ & $39(13.4)$ & 9.107 & 0.028 & 0.282 & 1.000 & -0.845 & 1.000 & 1.127 & 1.000 & 2.724 & 0.039 & -2.442 & 0.088 & -1.597 & 0.662 \\
\hline $\mathrm{BCM}, \mathrm{kg}$ & & $\begin{array}{c}29.8 \\
(10.25)\end{array}$ & $\begin{array}{c}29.7 \\
(12.17)\end{array}$ & $\begin{array}{l}29.83 \\
(10.92)\end{array}$ & 29.7 (10) & 13.823 & 0.006 & 1.409 & 0.953 & -2.667 & 0.045 & 1.268 & 1.000 & 2.020 & 0.261 & -3.429 & 0.004 & -0.751 & 1.000 \\
\hline $\mathrm{BMR}, \mathrm{kcal} / \mathrm{d}$ & & $\begin{array}{c}1,626 \\
(422.25)\end{array}$ & $\begin{array}{l}1,612 \\
(410.0)\end{array}$ & $\begin{array}{c}1,617 \\
(404.75)\end{array}$ & $\begin{array}{c}1,604 \\
(383.25)\end{array}$ & 23.01 & $<0.001$ & 4.496 & $<0.001$ & 3.411 & 0.001 & 1.958 & 0.05 & 2.043 & 0.041 & 1.983 & 0.047 & 0.128 & 0.898 \\
\hline $\mathrm{SBP}, \mathrm{mmHg}$ & & $120(25)$ & $120(21)$ & 115 (20) & $120(20)$ & 12.229 & 0.007 & 1.503 & 0.797 & -1.409 & 0.953 & 2.912 & 0.022 & 0.094 & 1.000 & -1.409 & 0.953 & -2.818 & 0.029 \\
\hline DBP, mmHg & & $80(10)$ & $70(10)$ & $70(10)$ & $75(10)$ & 13.246 & 0.004 & 2.959 & 0.019 & 0.000 & 1.000 & 2.959 & 0.019 & 2.160 & 0.184 & -0.798 & 1.000 & -0.798 & 1.000 \\
\hline Hematocrit & $0.395-0.505$ & $\begin{array}{c}0.416 \\
(0.036)\end{array}$ & $\begin{array}{c}0.40 \\
(0.05)\end{array}$ & $\begin{array}{c}0.41 \\
(0.03)\end{array}$ & $\begin{array}{c}0.408 \\
(0.042)\end{array}$ & 11.620 & 0.009 & 3.006 & 0.016 & -2.818 & 0.029 & 0.188 & 1.000 & 1.127 & 1.000 & -1.879 & 0.362 & -0.939 & 1.000 \\
\hline $\begin{array}{l}\text { Glucose, } \\
\mathrm{mg} / \mathrm{dl}\end{array}$ & $60-110$ & $\begin{array}{c}84.0 \\
(12.5)\end{array}$ & $\begin{array}{l}72.5 \\
(7.75)\end{array}$ & $\begin{array}{l}84.0 \\
(9.75)\end{array}$ & $\begin{array}{c}84.71 \\
(13.25)\end{array}$ & 43.45 & $<0.001$ & 4.59 & $<0.001$ & 4.915 & $<0.001$ & 0.658 & 0.51 & 1.325 & 0.185 & 4.958 & $<0.001$ & 0.932 & 0.351 \\
\hline $\begin{array}{l}\mathrm{HbA} 1 \mathrm{c} \text {, } \\
\mathrm{mmol} / \mathrm{mol} \mathrm{Hb}\end{array}$ & $<42.0$ & $33.3(4.4)$ & $\begin{array}{l}32.75 \\
(5.23)\end{array}$ & $\begin{array}{c}31.65 \\
(5.4)\end{array}$ & $\begin{array}{l}33.16 \\
(5.12)\end{array}$ & 48.92 & $<0.001$ & 1.222 & 0.222 & 4.838 & $<0.001$ & 4.471 & $<0.001$ & 3.368 & 0.001 & 3.069 & 0.002 & 4.428 & $<0.001$ \\
\hline $\begin{array}{l}\text { Fructosamine, } \\
\mu \mathrm{mol} / / \mathrm{l}\end{array}$ & $205-285$ & $\begin{array}{c}243.5 \\
(24)\end{array}$ & $253(32)$ & $248(22)$ & 248 (17) & 32.056 & $<0.001$ & 5.401 & $<0.001$ & -2.630 & 0.051 & 2.771 & 0.034 & 4.039 & $<0.001$ & -1.362 & 1.000 & -1.268 & 1.000 \\
\hline $\begin{array}{l}\text { 3-OH-B, } \\
\mathrm{mmol} / \mathrm{l}\end{array}$ & & $0.18(0.3)$ & $0.15(0.3)$ & $0.1(0.2)$ & $0(0.2)$ & 5.000 & 0.172 & & & & & & & & & & & & \\
\hline $\mathrm{HDL}, \mathrm{mg} / \mathrm{dl}$ & $>45$ & $58.5(25)$ & $58.5(19)$ & $58(23)$ & $56(17)$ & 10.495 & 0.015 & 0.094 & 1.000 & -0.047 & 1.000 & 0.141 & 1.000 & 2.489 & 0.077 & -2.583 & 0.059 & -2.630 & 0.051 \\
\hline LDL, mg/dl & $<130$ & $119(43)$ & $\begin{array}{c}115.5 \\
(47)\end{array}$ & $117(45)$ & 119 (33) & 2.063 & 0.559 & & & & & & & & & & & & \\
\hline $\begin{array}{l}\text { Creatinine, } \\
\mathrm{mg} / \mathrm{dl}\end{array}$ & $0.70-1.20$ & $\begin{array}{l}0.765 \\
(0.21)\end{array}$ & $\begin{array}{c}0.80 \\
(0.19)\end{array}$ & $\begin{array}{c}0.81 \\
(0.24)\end{array}$ & $\begin{array}{l}0.80 \\
(0.21)\end{array}$ & 7.410 & 0.060 & & & & & & & & & & & & \\
\hline $\begin{array}{l}\text { Cystatin C, } \\
\mathrm{mg} / \mathrm{l}\end{array}$ & $0.47-1.09$ & $0.88(0.2)$ & $\begin{array}{c}0.89 \\
(0.16)\end{array}$ & $0.9(0.14)$ & $\begin{array}{c}0.92 \\
(0.21)\end{array}$ & 6.960 & 0.073 & & & & & & & & & & & & \\
\hline $\begin{array}{l}\text { GFR } \\
\text { (CKD-EPI) }\end{array}$ & & $91(0)$ & $91(0)$ & 91 (1) & $91(36)$ & 1.943 & 0.584 & & & & & & & & & & & & \\
\hline $\mathrm{pNa}^{+}, \mathrm{mmol} / \mathrm{l}$ & $136-145$ & $141(36)$ & $142(36)$ & $142(36)$ & $141(3)$ & 3.884 & 0.274 & & & & & & & & & & & & \\
\hline $\mathrm{pK}^{+}, \mathrm{mmol} / \mathrm{l}$ & $3.4-4.5$ & $4.0(0.4)$ & $3.8(0.4)$ & $4.1(0.3)$ & $4.0(0.4)$ & 16.111 & 0.001 & 1.315 & 1.000 & -3.851 & 0.001 & 2.536 & 0.067 & 0.282 & 1.000 & -1.597 & 0.662 & -2.254 & 0.145 \\
\hline $\mathrm{cNa}^{+}, \mathrm{mmol} / \mathrm{l}$ & $136-145$ & $142(36)$ & $142(36)$ & $141.5(3)$ & 141 (3) & 6.683 & 0.083 & & & & & & & & & & & & \\
\hline $\mathrm{cK}^{+}, \mathrm{mmol} / \mathrm{l}$ & $3.3-5.1$ & $4.0(0.3)$ & $3.8(0.4)$ & $4.1(0.3)$ & $4.0(0.2)$ & 10.867 & 0.012 & 1.221 & 1.000 & -2.771 & 0.034 & 1.550 & 0.727 & 1.362 & 1.000 & -2.583 & 0.059 & -0.188 & 1.000 \\
\hline
\end{tabular}



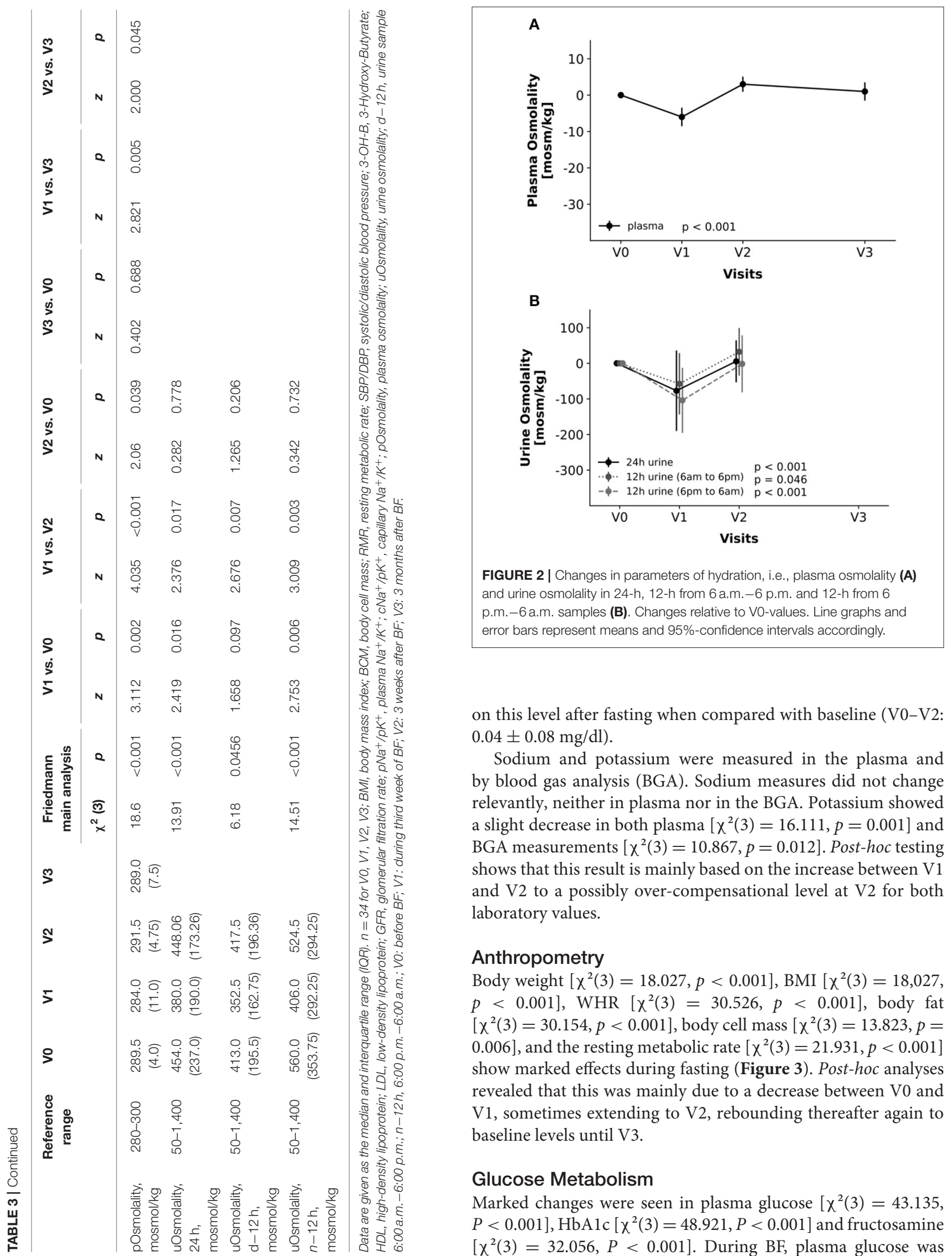

on this level after fasting when compared with baseline (V0-V2: $0.04 \pm 0.08 \mathrm{mg} / \mathrm{dl}$ ).

Sodium and potassium were measured in the plasma and by blood gas analysis (BGA). Sodium measures did not change relevantly, neither in plasma nor in the BGA. Potassium showed a slight decrease in both plasma $\left[\chi^{2}(3)=16.111, p=0.001\right]$ and BGA measurements $\left[\chi^{2}(3)=10.867, p=0.012\right]$. Post-hoc testing shows that this result is mainly based on the increase between V1 and V2 to a possibly over-compensational level at V2 for both laboratory values.

\section{Anthropometry}

Body weight $\left[\chi^{2}(3)=18.027, p<0.001\right]$, BMI $\left[\chi^{2}(3)=18,027\right.$, $p<0.001]$, WHR $\left[\chi^{2}(3)=30.526, p<0.001\right]$, body fat $\left[\chi^{2}(3)=30.154, p<0.001\right]$, body cell mass $\left[\chi^{2}(3)=13.823, p=\right.$ $0.006]$, and the resting metabolic rate $\left[\chi^{2}(3)=21.931, p<0.001\right]$ show marked effects during fasting (Figure 3). Post-hoc analyses revealed that this was mainly due to a decrease between V0 and $\mathrm{V} 1$, sometimes extending to $\mathrm{V} 2$, rebounding thereafter again to baseline levels until V3.

\section{Glucose Metabolism}

Marked changes were seen in plasma glucose $\left[\chi^{2}(3)=43.135\right.$, $P<0.001]$, HbAlc $\left[\chi^{2}(3)=48.921, P<0.001\right]$ and fructosamine $\left[\chi^{2}(3)=32.056, P<0.001\right]$. During $B F$, plasma glucose was 
A

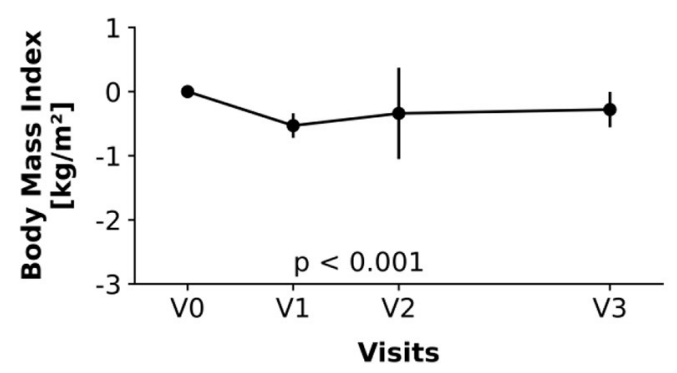

B

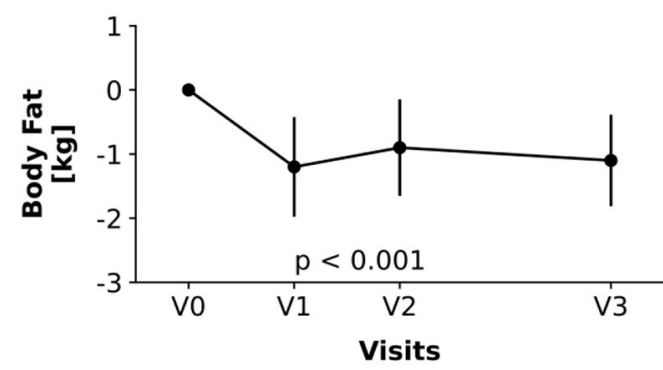

C

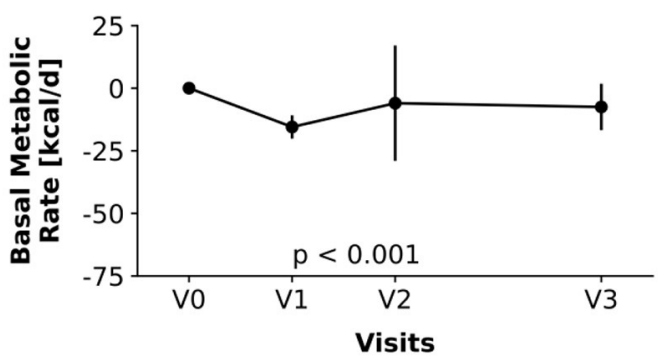

D

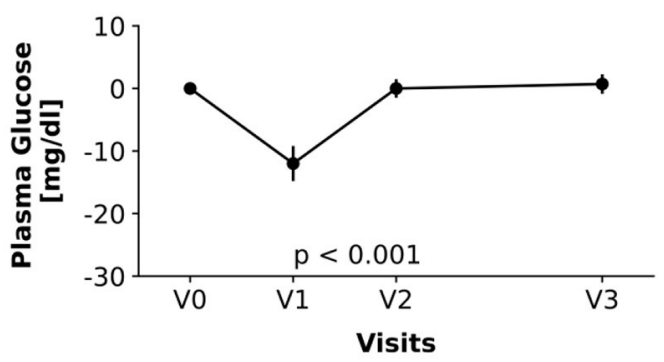

E

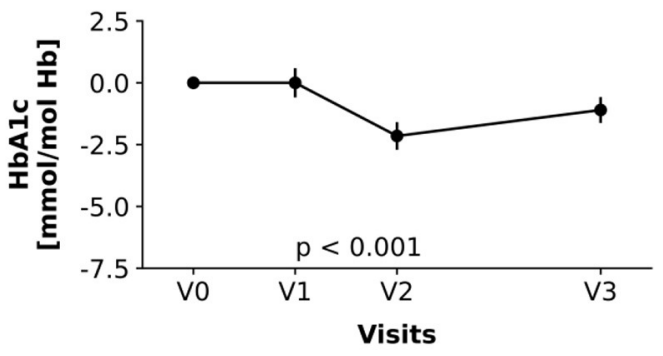

FIGURE 3 | Changes in metabolic parameters over the study period (A: BMI, B: body fat mass, C: resting metabolic rate, D: glucose, and $\mathbf{E}: \mathrm{HbA1C}$. Changes relative to VO-values. Line graphs and error bars represent means and 95\%-confidence intervals accordingly.
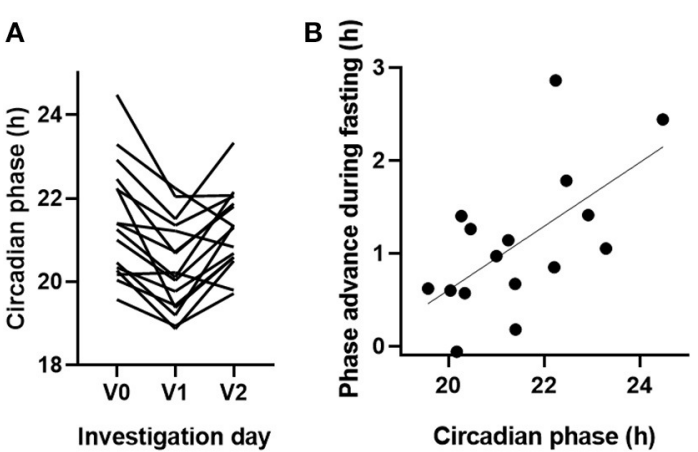

FIGURE 4 | Bahá'í fasting advanced the circadian phase in particular of late chronotypes. (A) The circadian phase of 16 study participants was assessed, using the blood monocyte-based BodyTime assay (26) before (V0), during (V1), and 3 weeks after BF (V2). The circadian phase corresponds to the BodyTime-predicted DLMO, which usually occurs about 2 hours before habitual bedtime. (B) The extent of advancing the circadian phase during BF correlated with chronotype (i.e., the circadian phase before BF).

considerably lower compared with visits before and after the fast. Interestingly, $\mathrm{HbAlc}$ was decreased with a temporal delay to the fast, with a smaller decrease between $\mathrm{V} 0$ and $\mathrm{V} 1$, reaching the minimum only at V2. Here, even values at V3 were lower when compared with baseline values.

Similarly, fructosamine was elevated during and after the fast (V1, V2, and V3 vs. V0), indicating only slow recovery.

\section{Chronobiology}

The circadian phase (chronotype) of a subject, given by the predicted dim-light melatonin onset (DLMO) assessed, using the blood monocyte-based assay BodyTime (26), varied markedly between the days of investigation $\left[\chi^{2}(2)=13.5, P=0.001\right]$. Chronotype during fasting (V1) was notably earlier than in V0 but returned to pre-fasting levels within the next 3 weeks (V2). The extent of advancing the circadian phase correlated distinctly with the initial chronotype, i.e., late chronotypes advanced more than early chronotypes (slope $=0.34, P=0.012, r=0.61$, Figure 4). No correlations with other clinical outcomes were found after adjustment.

\section{Sex Effects}

Repeated-measures ANOVA was used to examine the changes over time in the interaction between sex and visits for BMI, body fat mass, resting metabolic rate, glucose, HbA1c, plasma, and urine osmolality (including 24-hour, diurnal and nocturnal measurements), as well as for the chronobiological data. In this explorative analysis, we could not detect any relevant differences.

\section{DISCUSSION}

This study aimed to generate hypotheses about the health effects and safety of religiously motivated diurnal IDF. Particular emphasis was placed on changes of hydration status, as this aspect is the least scientifically explored until now. Our results showed that hydration status varied slightly 
between fasting and non-fasting times in this study sample. Although the mean changes in osmolality were within the physiological range, it could, on scrutiny, be observed that some dynamics seem to be present during nighttime, while diurnal samples remain almost unchanged compared with nonfasting samples.

In summary, our data indicate the safety of BF regarding hydration and renal function. Even a slight diluting effect on urine and plasma was observed. Despite a lower resting metabolic rate, the anthropometric indices, as well as glucose metabolism, seemed to profit from BF, without a rebound being witnessed even 3 months later. The phase of circadian rhythms was changed during BF, an effect being caused either by fasting itself or by concomitant behavioral changes.

To our knowledge, this study is the first worldwide to assess physiological and psychological changes during the religious fast of the Bahá ís. Different methodological approaches were chosen to unravel cross-links between physiological parameters and religious experience and objective and subjective dimensions of this specific fast. This approach was confirmed even in interpreting the laboratory data on the main study focus, which was the hydration status. We showed that, during $\mathrm{BF}$, hydration parameters remained within physiological limits or even indicated a dilution. Both serum and 24-hour-urine osmolality dropped. In the qualitative interviews, some subjects explained they would more consciously drink in the mornings before sunrise (own data, publication pending), which could explain part of the effect. But, as serum osmolality during BF was measured in the afternoon, there might be more effects leading to the slight dilution observed in most subjects. One of these could be activation of the renin-angiotensin-aldosterone system. The RAAS preserves body water resources by retaining sodium and eliminating potassium. According to our data, the latter actually decreased during BF, a finding which is in line with those in Ramadan fasting $(31,32)$. This would also suit the slight increase in osmolality in the afternoon spot urine samples. In prolonged DF, an increase in RAAS activity has been described (20), as well as an increase in serum osmolality only after 24-hour of DF, while urine volume decreased (15). Our data showed a decrease in both serum and urine osmolality in the 12- and 24-hour samples, while total body water remained unchanged in the bioimpedance analysis. Our findings suggest that, in the population studied, no relevant dehydration occurred during IDF.

Comparing the outcomes of $\mathrm{BF}$ on body weight and the metabolic rate with the results from the studies on Ramadan fasting, we see a diverse picture in Ramadan fasting. Some studies report a weight loss, others no change and even others a weight gain during Ramadan fasting (33). Also, it seems that mostly overweight people lose weight in Ramadan, while those with normal weight do not show as much effect (12). As not enough overweight individuals with a BMI $\geq 25 \mathrm{~kg} / \mathrm{m}^{2}$ were included, this study cannot confirm a similar tendency in BF. A first data analysis of our sub-study at the ECRC indicated an increased adipose tissue lipid mobilization (publication pending). Thus, it could be postulated that BF triggers lipid metabolism, leading to a loss of body fat mass and body weight despite a reduction of the resting metabolic rate.
Furthermore, the observed weight loss and the drop of the resting metabolic rate, alongside the measurement of the activity of the clock genes, show that cyclical changes in energy metabolism influence nutrient utilization and that shortterm changes in meal frequency and timing have an effect on chronobiology and energy balance. This is in line with the findings on Ramadan fasting (33).

Previous studies showed that intermittent fasting can affect sleep-wake patterns as well as circadian rhythms in animals and humans. Popular readouts for circadian rhythmicity are repeated measures of body temperature and melatonin levels as well as subjective assessment of chronotype, using questionnaires. Here, a recently developed BodyTime assay was used, in which only one blood sample is needed to objectively assess the phase of the circadian clock of an individual (26). Although the BodyTime assay has not been validated in individuals under specific fasting dietary regimens, it is important to note that, in the study, in which we identified the biomarkers (26), the subjects received hourly isocaloric snacks, whereas, in the subsequent validation study, the participants were free to choose the dietary regimen. At least, when comparing these largely different dietary regimens, we found no difference in accuracy in the circadian phase assessment. The results showed that BF markedly advanced the circadian phase of fasting individuals by more than 1 hour, which is reversed to normal levels 3 weeks after fasting (Figure 4A). In animals, restricting food intake to a few hours within their inactive phase substantially alters circadian rhythms in peripheral tissues, while the master clock in the hypothalamic suprachiasmatic nucleus remains largely unaffected [for a review, see Manoogian and Panda (34)]. Studies investigating how Ramadan fasting affects circadian rhythms revealed conflicting results, since they often cannot discriminate direct effects of fasting on the circadian system from indirect effects of lifestyle changes [for a review, see Qasrawi et al. (35)]. However, it remains unclear whether BF alters the circadian system per se or whether the observed phase advance of circadian rhythms is rather due to altered sleeping times and thus altered light exposure, which would phase shift circadian rhythms. A limitation of our study is that we recorded neither light levels nor sleeping time, which would have probably been helpful to discriminate between these hypotheses. However, the fact that we observed larger phase advances for later chronotypes (Figure 4B) supports the second hypothesis, since the participants reported eating and drinking before sunrise (own data, publication pending), which requires relatively earlier wake-up times for late types during $\mathrm{BF}$ compared with early types.

A possible bias of the study may originate from the fact that rather healthy, highly educated volunteers participated in this study. This sociodemographic distribution reflects findings from another sample of Bahá ís in Germany (21). To counteract these biases and to track intraindividual changes during BF, this study was designed as a longitudinal study with a pretest and a longer follow-up than most of the Ramadan studies. As, in Germany, the Bahái i religion only has $\sim 6,000$ followers in more than 100 localities (21), it was a challenge to get an adequate sample size. Future confirmatory studies should be conducted in areas with more Baháís to allow higher participant numbers. Timing 
of samplings was yet another challenge. During BF, fasting measurements were not possible in the mornings, as breakfast was taken at dawn. Changes seen in physiological parameters during BF may thus include time-of-day effects. To meet this challenge, we included longer-term parameters, for example, 24 h-urine samples for osmolality or $\mathrm{HbAlc}$ for serum glucose, as well as follow-up measurements. These show longer-lasting changes, so we can exclude these were only due to time-ofday effects.

Studies with higher participant numbers are needed to validate the hypothesis of urine dilution and weight loss during BF, as well as the improvement of glucose and fat metabolism. Future studies should also focus on exploring the physiological background of these clinical changes and their potential impact on long-term health. It should also be considered to study BF in different cultural contexts, as Baháís fast all over the world. Culturally different approaches to fasting as well as the meal composition could influence the outcomes under discussion.

In summary, this study showed that Bahái fasting, although being shorter than other intermittent fasting regimes (5), might have a notable impact on fat and glucose metabolism. Refraining from drinking as well as chronobiological considerations might be crucial in this metabolic shift and should be studied further, especially as this study has not found critical effects on hydration or renal parameters in this kind of intermittent dry fasting.

\section{DATA AVAILABILITY STATEMENT}

The original data of the chronobiological measurements can be found in Supplementary Material. Futher data described in the article will be made available upon request from the corresponding author pending application and approval.

\section{ETHICS STATEMENT}

The study protocol was approved by the institutional review board of Charité Universitätsmedizin Berlin (Charitéplatz 1, 10117 Berlin) in January 2018 (ID: EA4/216/17) and

\section{REFERENCES}

1. Di Francesco A, Di Germanio C, Bernier M, de Cabo R. A time to fast. Science. (2018) 362:770-5. doi: 10.1126/science.aau2095

2. Longo VD, Mattson MP. Fasting: molecular mechanisms and clinical applications. Cell Metab. (2014) 19:181-92. doi: 10.1016/j.cmet.2013.12.008

3. Brandhorst S, Choi IY, Wei M, Cheng CW, Sedrakyan S, Navarrete G, et al. A periodic diet that mimics fasting promotes multi-system regeneration, enhanced cognitive performance, and healthspan. Cell Metab. (2015) 22:8699. doi: 10.1016/j.cmet.2015.05.012

4. Longo VD, Panda S. Fasting, circadian rhythms, and timerestricted feeding in healthy lifespan. Cell Metab. (2016) 23:1048-59. doi: 10.1016/j.cmet.2016.06.001

5. Mattson MP, Longo VD, Harvie M. Impact of intermittent fasting on health and disease processes. Ageing Res Rev. (2017) 39:46-58. doi: 10.1016/j.arr.2016.10.005

6. Rangan P, Choi I, Wei M, Navarrete G, Guen E, Brandhorst S, et al. FastingMimicking diet modulates microbiota and promotes intestinal regeneration to was registered with clinical trials (ID: NCT03443739). The participants provided their written informed consent to participate in this study.

\section{AUTHOR CONTRIBUTIONS}

DK-L conceived and designed the study and had primary responsibility for final content. $\mathrm{MB}$ and $\mathrm{AMä}$ assisted in designing the part of the metabolic measurements. AK designed the chronobiological part of the study. DK-L, CKl, JS, RR, AMä, $\mathrm{MB}, \mathrm{BK}$, and $\mathrm{MJ}$ conducted the research. DK-L, CKl, FK, NS, JS, AMä, MB, CKe, RR, AMi, BA, and AK analyzed and interpreted data. DK-L, CKl, FK, AK, AMi, MB, and NS wrote the paper. All the authors have read and approved the final manuscript.

\section{FUNDING}

The study was funded by the German Baháí Research Fund (Stiftung für Baháí Studien: Vorstand Dr. Nicola Towfigh, Nordstrasse 18, 48149 Münster), with a sum of 27,105 EUR. The funders were not involved in designing or executing any part of the present study.

\section{ACKNOWLEDGMENTS}

We thank Miriam Rösner (Institute of Social Medicine, Epidemiology and Health Economics, CharitéUniversitätsmedizin Berlin) and Gabriele Rahn (Experimental and Clinical Research Center, Charité-Universitätsmedizin Berlin) for assisting in screening the study participants and patient care during clinical protocols. We also thank Dr. Dimitra Varvarezou for carefully proofreading the manuscript as a native speaker.

\section{SUPPLEMENTARY MATERIAL}

The Supplementary Material for this article can be found online at: https://www.frontiersin.org/articles/10.3389/fnut.2021. 662310/full\#supplementary-material reduce inflammatory bowel disease pathology. Cell Rep. (2019) 26:2704-19.e6. doi: 10.1016/j.celrep.2019.02.019

7. Michalsen A. Prolonged fasting as a method of mood enhancement in chronic pain syndromes: a review of clinical evidence and mechanisms. Curr Pain Headache Rep. (2010) 14:80-7. doi: 10.1007/s11916-010-0104-Z

8. Wilhelmi de Toledo F, Buchinger A, Burggrabe H, Holz G, Kuhn C, Lischka E, et al. Fasting therapy - an expert panel update of the 2002 consensus guidelines. Forsch Komplementmed. (2013) 20:434-43. doi: $10.1159 / 000357602$

9. Li C, Sadraie B, Steckhan N, Kessler C, Stange R, Jeitler M, et al. Effects of A one-week fasting therapy in patients with type-2 diabetes mellitus and metabolic syndrome - a randomized controlled explorative study. Exp Clin Endocrinol Diabetes. (2017) 125:618-24. doi: 10.1055/s-0043-10 1700

10. Bauersfeld SP, Kessler CS, Wischnewsky M, Jaensch A, Steckhan N, Stange $\mathrm{R}$, et al. The effects of short-term fasting on quality of life and tolerance to chemotherapy in patients with breast and ovarian cancer: a randomized crossover pilot study. BMC Cancer. (2018) 18:476. doi: 10.1186/s12885-018-4353-2 
11. Faris MAE, Jahrami HA, Alsibai J, Obaideen AA. Impact of ramadan diurnal intermittent fasting on metabolic syndrome components in healthy, nonathletic muslim people aged over 15 years: a systematic review and metaanalysis. Br J Nutr. (2019). doi: 10.1017/S000711451900254X. [Epub ahead of print].

12. Fernando HA, Zibellini J, Harris RA, Seimon RV, Sainsbury A. Effect of ramadan fasting on weight and body composition in healthy non-athlete adults: a systematic review and meta-analysis. Nutrients. (2019) 11:478. doi: 10.3390/nu11020478

13. Roumelioti ME, Glew RH, Khitan ZJ, Rondon-Berrios H, Argyropoulos CP, Malhotra D, et al. Fluid balance concepts in medicine: Principles and practice. World J Nephrol. (2018) 7:1-28. doi: 10.5527/wjn.v7.i1.1

14. Persynaki A, Karras S, Pichard C. Unraveling the metabolic health benefits of fasting related to religious beliefs: A narrative review. Nutrition. (2017) 35:14-20. doi: 10.1016/j.nut.2016.10.005

15. Papagiannopoulos IA, Sideris VI, Boschmann M, Koutsoni OS, Dotsika EN. Anthropometric, hemodynamic, metabolic, and renal responses during 5 days of food and water deprivation. Forsch Komplementmed. (2013) 20:427-33. doi: 10.1159/000357718

16. Horne BD, May HT, Anderson JL, Kfoury AG, Bailey BM, McClure BS, et al. Usefulness of routine periodic fasting to lower risk of coronary artery disease in patients undergoing coronary angiography. Am J Cardiol. (2008) 102:814-9. doi: 10.1016/j.amjcard.2008.05.021

17. Mosek A, Korczyn AD. Yom kippur headache. Neurology. (1995) 45:1953-5. doi: 10.1212/WNL.45.11.1953

18. Kundin JE. Yom kippur headache. Neurology. (1996) 47:854. doi: 10.1212/WNL.47.3.854

19. Grajower MM. Management of diabetes mellitus on yom kippur and other jewish fast days. Endocr Pract. (2008) 14:305-11. doi: 10.4158/EP.14.3.305

20. Papagiannopoulos-Vatopaidinos IE, Papagiannopoulou M, Sideris V. Dry fasting physiology: responses to hypovolemia and hypertonicity. Complement Med Res. (2020) 27:242-51. doi: 10.1159/000505201

21. Demmrich S. How to measure baha'i religiosity: the crsi-20 for baha'is as a first reliable and valid measurement. Religions. (2020) 11:29. doi: $10.3390 /$ rel11010029

22. Baháu'lláh. The Kitáb-i-Aqdas: The Most Holy Book. 1 ed. London: Baha'i Publishing Trust (1993). 315p.

23. BaHammam AS, Almeneessier AS. Recent evidence on the impact of ramadan diurnal intermittent fasting, mealtime, and circadian rhythm on cardiometabolic risk: a review. Front Nutr. (2020) 7:28. doi: $10.3389 /$ fnut.2020.00028

24. Shadish WR CT, Campbell DT. Quasi-Experimentational Designs that Either Lack a Control Group or Lack Pretest Observations on the Outcome. Boston, MA: Houghton Mifflin (2002).

25. Badanta-Romero B, de Diego-Cordero R, Rivilla-Garcia E. Influence of religious and spiritual elements on adherence to pharmacological treatment. J Relig Health. (2018) 57:1905-17. doi: 10.1007/s10943-018-0606-2

26. Wittenbrink N, Ananthasubramaniam B, Munch M, Koller B, Maier B, Weschke C, et al. High-accuracy determination of internal circadian time from a single blood sample. J Clin Invest. (2018) 128:3826-39. doi: 10.1172/JCI120874

27. Roenneberg T, Wirz-Justice A, Merrow M. Life between clocks: daily temporal patterns of human chronotypes. J Biol Rhythms. (2003) 18:80-90. doi: $10.1177 / 0748730402239679$

28. Cohen J. Statistical Power Analysis for the Behavioral Sciences. 2 ed. Hillsdale, NJ: Erlbaum (1988).

29. Mitchell BD, McArdle PF, Shen H, Rampersaud E, Pollin TI, Bielak LF, et al. The genetic response to short-term interventions affecting cardiovascular function: rationale and design of the heredity and phenotype intervention (HAPI) heart study. Am Heart J. (2008) 155:823-8. doi: 10.1016/j.ahj.2008.01.019

30. Bundesamt S. Health Status, Behaviour Relevant to Health 2020 (2020). Available online at: https://www.destatis.de/EN/Themes/SocietyEnvironment/Health/Health-Status-Behaviour-Relevant-Health/_node. html;sessionid=FF4C4A03AB2281D1B970D17186A4E6C3.internet711

31. Attarzadeh Hosseini SR, Hejazi K. The effects of ramadan fasting and physical activity on blood hematological-biochemical parameters. Iran J Basic Med Sci. (2013) 16:845-9.

32. Miladipour AH, Shakhssalim N, Parvin M, Azadvari M. Effect of ramadan fasting on urinary risk factors for calculus formation. Iran J Kidney Dis. (2012) 6:33-8.

33. Lessan N, Ali T. Energy metabolism and intermittent fasting: the ramadan perspective. Nutrients. (2019) 11:1192. doi: 10.3390/nu11051192

34. Manoogian ENC, Panda S. Circadian rhythms, time-restricted feeding, and healthy aging. Ageing Res Rev. (2017) 39:59-67. doi: 10.1016/j.arr.2016.12.006

35. Qasrawi SO, Pandi-Perumal SR, BaHammam AS. The effect of intermittent fasting during ramadan on sleep, sleepiness, cognitive function, and circadian rhythm. Sleep Breath. (2017) 21:577-86. doi: 10.1007/s11325-017-1473-x

Conflict of Interest: The authors declare that the research was conducted in the absence of any commercial or financial relationships that could be construed as a potential conflict of interest.

Publisher's Note: All claims expressed in this article are solely those of the authors and do not necessarily represent those of their affiliated organizations, or those of the publisher, the editors and the reviewers. Any product that may be evaluated in this article, or claim that may be made by its manufacturer, is not guaranteed or endorsed by the publisher.

Copyright (c) 2021 Koppold-Liebscher, Klatte, Demmrich, Schwarz, Kandil, Steckhan, Ring, Kessler, Jeitler, Koller, Ananthasubramaniam, Eisenmann, Mähler, Boschmann, Kramer and Michalsen. This is an open-access article distributed under the terms of the Creative Commons Attribution License (CC BY). The use, distribution or reproduction in other forums is permitted, provided the original author(s) and the copyright owner(s) are credited and that the original publication in this journal is cited, in accordance with accepted academic practice. No use, distribution or reproduction is permitted which does not comply with these terms. 